\title{
Identified Ankle Extensor and Flexor Motoneurons Display Different Firing Profiles in the Neonatal Rat
}

\author{
Florence Cotel, ${ }^{1}$ Myriam Antri, ${ }^{2}$ Jean-Yves Barthe, ${ }^{1}$ and Didier Orsal ${ }^{1}$ \\ ${ }^{1}$ Laboratoire de Neurobiologie des Signaux Intercellulaires, Centre National de la Recherche Scientifique Unité Mixte de Recherche (CNRS UMR) 7101, \\ Université Pierre et Marie Curie-Paris 6, F-75252 Paris Cedex 05, France, and 2Laboratoire Motricité, Adaptation, Cognition, CNRS UMR 5227, Université \\ Bordeaux 2, F-33076 Bordeaux Cedex, France
}

The present study was designed to compare the firing profiles exhibited by lumbar flexor or extensor motoneurons in response to injection of depolarizing/repolarizing currents. Motoneurons were recorded intracellularly in the in vitro brainstem-spinal cord of newborn rats (P4-P7). They were synaptically isolated and identified by antidromic stimulations of the central stump of flexor or extensor muscle nerves: tibialis anterior (ankle flexor) and gastrocnemius medialis or lateralis (ankle extensors). Two protocols were applied to establish the four firing profiles previously described (type I-IV) (Bennett et al., 2001): (1) symmetric depolarizing/repolarizing ramps of current and (2) progressive steps of depolarizing currents followed by equivalent steps of repolarizing current. According to such profiles, this study clearly shows that flexor and extensor motoneurons are different. The whole population of flexor motoneurons solely exhibited the type II profile, characterized by a frequency-current $(F-I)$ relationship with a clockwise hysteresis. In contrast, in addition to this type II profile, the other three profiles of repetitive firing (type I, III and IV) were observed in extensor motoneurons; a linear $F-I$ relationship (type I profile), a self-sustained discharge pattern together with a linear $F-I$ relationship (type III profile) and a self-sustained firing pattern together with an $F-I$ relationship showing a counter-clockwise hysteresis (type IV profile). Thus, during the early postnatal development, a significant part of the population of extensor motoneurons, but not flexors, are able to produce selfsustained discharges known to involve the activation of persistent inward currents.

\section{Introduction}

Locomotion is an automatic, repetitive and perfectly well structured rhythmic motor activity. Typically, the locomotor cycle is divided into the swing and the stance phases. A common finding is that the duration of each phase is differently correlated to the duration of the whole step cycle (Halbertsma, 1983). This difference is generally attributed to different wirings in premotoneuronal networks controlling either flexor or extensor motoneurons (respectively F-MNs and E-MNs). However, an alternative hypothesis proposes a possible role of intrinsic membrane properties, such as plateau potentials or self-sustained firing, in regulating the duration of motoneuronal firing (Kiehn and Eken, 1998), where F-MNs and E-MNs could express different intrinsic properties.

A plateau potential is a long-lasting depolarization that can be experimentally induced by a brief pulse of depolarizing current (Russell and Hartline, 1978). This property has been described in motoneurons from various species [cat (Hounsgaard et al.,

\footnotetext{
Received June 23, 2008; revised 0ct. 22, 2008; accepted Jan. 20, 2009.

F.C. received a stipend from Institut pour la Recherche sur la Moelle épinière et l'Encéphale/Fondation pour la Recherche Médicale and from Agence Nationale pour la Recherche. We are indebted to Dr. Laurent Vinay for the dissection of the newborn spinal cord preserving the lumbar plexus and express our gratitude to K. Fenelon for her valuable comments.

Correspondence should be addressed to Dr. Didier Orsal, Laboratoire de Neurobiologie des Signaux Intercellulaires, CNRS UMR 7101, Université Pierre et Marie Curie-PARIS 6, 7 quai Saint-Bernard, F-75252 Paris Cedex 05, France. E-mail: orsal.didier@snv.jussieu.fr.

DOI:10.1523/JNEUROSCI.3462-08.2009

Copyright $\odot 2009$ Society for Neuroscience $\quad$ 0270-6474/09/292748-06\$15.00/0
}

1988b; Bennett et al., 1998a,b; Lee and Heckman 1998a,b), turtle (Hounsgaard et al., 1988a), rat (Bennett et al., 2001)]. In these species, plateaus were shown to depend on voltage-dependent $\mathrm{Ca}^{2+}$ or $\mathrm{Na}^{+}$conductances that do not deactivate or deactivate very slowly, allowing persistent inward currents (PICs) (Hounsgaard and Kiehn, 1989; Zhong et al., 2007). In general, neurons that possess the capability to generate PICs exhibit the following experimental characteristics: (1) the relationship between the intensity of a depolarizing current and the subsequent frequency of firing ( $F-I$ relationship) shows a counter-clockwise hysteresis (Lee and Heckman, 1998a,b; Button et al., 2006), and (2) the intensity of the current that initiates rhythmic firing is higher than the one that ends it. In the end, four typical profiles of firing pattern were described in response to ramps of depolarizing/ repolarizing current in sacral (Bennett et al., 2001) and lumbar (Button et al., 2006) motoneurons of chronic spinal rats. Types III and IV profiles correspond to neurons able to generate PICs. During locomotion, flexor and extensor muscles exhibit different duration in emg bursts, probably because intrinsic active properties could contribute to control the duration of motoneuronal firing.

Thus, the aim of our study was to correlate the firing profile of a given motoneuron to the function of the muscle it innervates. We have recorded identified and synaptically isolated lumbar motoneurons innervating either the triceps surae (extensor) or the tibialis anterior (flexor) muscles in in vitro spinal cord of newborn rats (P4-P7). Progressive depolarization/repolarization steps and triangular shaped current ramps were used to in- 
Table 1. Summary of F-MN and E-MN properties

\begin{tabular}{lccc}
\hline Motoneuron properties & Flexor & Extensor & $p_{0.95}$ \\
\hline Spike amplitude (mV) & $79.0 \pm 1.4(19)$ & $86.3 \pm 1.8(21)$ & $p<0.01$ \\
Spike width (ms) & $1.3 \pm 0.3(18)$ & $1.2 \pm 0.2(21)$ & NS \\
AHP amplitude (mV) & $1.7 \pm 1.5(14)$ & $3.6 \pm 1.5(13)$ & $p<0.01$ \\
AHP half-decay time (ms) & $31.8 \pm 3.4(14)$ & $36.1 \pm 9.9(14)$ & NS \\
Rheobase (nA) & $1.3 \pm 0.1(20)$ & $1.6 \pm 0.1(23)$ & NS \\
Input resistance (MS) & $20.8 \pm 1.9(16)$ & $19.9 \pm 1.9(19)$ & NS \\
ePIC (nA) & $0.46 \pm 0.04(20)$ & $0.05 \pm 0.02(23)$ & $p<0.001$ \\
F-Islope (Hz/nA) & $20.4 \pm 0.8(20)$ & $12.3 \pm 0.8(23)$ & $p<0.001$ \\
\hline
\end{tabular}

Values are means \pm SEM. Number of motoneurons is indicated in parentheses. NS, Not significant, unpaired $t$ test.

vestigate the voltage-dependent self-sustained firing of motoneurons. Our results show for the first time that $100 \%$ of F-MNs demonstrate early derecruitment, whereas $30 \%$ of E-MNs are already able to generate PICs.

\section{Materials and Methods}

Dissection. The experiments were performed on 20 newborn rats from $\mathrm{P} 4$ to P7 (postnatal day). Experimental procedures were made to minimize animal suffering, in accordance with the guidelines of the European Community Council (86/609/EEC), French Ministry for Agriculture and local ethics committee of the Université Pierre et Marie Curie. The experimental procedure used to prepare the spinal cord has previously been described by Pflieger et al. (2002). Briefly, the animals were anesthetized with isoflurane (Forene, Abott), decapitated and eviscerated in oxygenated saline bubbled with $95 \% \mathrm{O}_{2}-5 \% \mathrm{CO}_{2}$ mixture with the following composition (in mM): $\mathrm{NaCl}: 130, \mathrm{NaH}_{2} \mathrm{PO}_{4}: 0.58, \mathrm{MgSO}_{4}: 1.3, \mathrm{CaCl}_{2}$ : $3.75, \mathrm{NaHCO}_{3}: 25$, D-glucose: $10, \mathrm{KCl}: 4 ; \mathrm{pH}$ 7.4. Brainstem, spinal cord and nerve branches innervating tibialis anterior (ankle flexor) and gastrocnemius medialis/lateralis (ankle extensor) muscles were carefully dissected and pinned down in the recording chamber, ventral side up.

Stimulation and recordings. Bipolar stainless electrodes were placed in contact with the three nerve branches and insulated with Vaseline. Motoneurons were identified as flexor $(n=20)$ or extensor $(n=23)$ when they demonstrated an antidromic spike in response to the corresponding muscle nerve stimulation $(0.5 \mathrm{~ms} ; 0.2-10 \mathrm{~mA}$; Master-8, A.M.P.I.; Isoflex, A.M.P.I.). Borosilicate microelectrodes (GC 100F-10 Harvard Apparatus) filled with $2 \mathrm{M} \mathrm{K}$-acetate $(40-80 \mathrm{M} \Omega$ ) were used for intracellular recordings of motoneurons $(n=43)$. Only motoneurons with a stable resting membrane potential more hyperpolarized than $-60 \mathrm{mV}$ and antidromic action potential with amplitudes of $>60 \mathrm{mV}$ (truncated in the figures) were included in the analysis. Intracellular recordings were made using Axoclamp 2B amplifier in bridge mode (Axon Instruments) connected to a computer via a Digidata 1200 interface (Axon Instruments). The intracellular signals were digitized $(2-10 \mathrm{kHz})$ using Clampex 7 software (pCLAMP7 pack program; Axon Instruments). In all the experiments, strychnine, APV and CNQX (10 $\mu \mathrm{m}$ each, Sigma) were added to the saline solution to block fast synaptic activities.

Protocols and data analysis. Depolarizing current pulses of incremental amplitude (700 ms, 0.1-5 nA) were used to determine the Rheobase (minimum depolarizing current intensity needed to generate a spike). Input resistance was measured by injecting square pulses of current $(-0.1 \mathrm{nA} ; 500 \mathrm{~ms}$; average of 10 trials). The amplitude and width of antidromic spikes (determined at half-amplitude of the spike) were measured. The amplitude and half-decay time of the AHP were also measured on antidromic spikes and averaged from at least 5 trials.

Progressive current steps ( $1 \mathrm{~s}$ duration each, total of $7 \mathrm{~s}$ ) allowed us to display three firing profiles: a symmetrical profile (current steps for recruitment and derecruitment were of equal intensity), an earlyderecruitment profile and a self-sustained firing profile. To classify the firing responses into the four types of firing profiles ("type I-IV profile" in the text) described by Bennett et al. (2001), the $F-I$ hysteresis of the firing responses was investigated by intracellular injection of progressive depolarizing/repolarizing triangular shaped current ramps ( $3.5 \mathrm{~s}$ depolarization followed by $3.5 \mathrm{~s}$ repolarization). For each protocol, $2-3$ trials were achieved for consistency purposes, and the interval between succes- sive trials was kept to $>10 \mathrm{~s}$. The slope of the current ramps ranged from 0.29 to 1.86 $\mathrm{nA} \cdot \mathrm{s}^{-1}$ and the amplitude of the current intensity ranged from $1.5 \times$ to $2 \times$ the rheobase. These protocols were used with and without bias current. However, because bias current did not modify the results, the figures illustrate experiments without bias current injection.

$F-I$ relationship was studied by measuring (1) the $F-I$ slope: (the slope on the depolarizing part of the protocol); (2) the estimated PIC (ePIC, difference in injected current at spike recruitment compared with spike derecruitment).

Unpaired $t$ tests were used to compare the antidromic spike amplitude and the width, the AHP amplitude and the half-decay time, the rheobase and the membrane resistance between E-MNs and F-MNs. A one-way ANOVA, followed by a Tukey's test for post hoc analysis was used to compare means between more than two groups. A confidence level of $p<0.05$ was considered statistically significant. Statistical analyses were performed on Graph pad prism software (GraphPad) and Origin 7.5 software (OriginLab). The data are indicated as the mean \pm SEM.

A quality control calculation was performed to determine the probability to record by random $X$ no-PIC-expressing motoneurons among a population of $Y$ containing $Z$ PIC-expressing motoneurons (Rozanov, 1936) (see Results).

\section{Results}

\section{General characteristics of recorded motoneurons}

The first step of this study was to compare the antidromic spike properties (the amplitude and the width), the AHP (the amplitude and the half-decay time), the input resistance and the rheobase of F-MNs and E-MNs (Table 1). Statistical analysis showed a significant difference in the amplitude of antidromic spike (mean value of F-MNs $79.0 \pm 1.4 \mathrm{mV}$ and mean value of E-MNs $86.3 \pm$ $1.8 \mathrm{mV} ; p<0.01$, unpaired $t$ test) and the amplitude of the AHP (mean value of F-MNs $1.7 \pm 1.5 \mathrm{mV}$ and mean value of E-MNs $3.6 \pm 1.5 \mathrm{mV} ; p<0.01$, unpaired $t$ test). No significant difference was measured between F-MNs and E-MNs regarding the spike width (mean value of F-MNs: $1.3 \pm 0.06$ and mean value of E-MNs: $1.2 \pm 0.03 \mathrm{~ms}$ ), the half-decay time of the AHP (mean value of F-MNs: $31.8 \pm 3.4 \mathrm{~ms}$ and mean value of E-MNs: $36.1 \pm$ $9.9 \mathrm{~ms}$ ), the rheobase (mean value of F-MNs: $1.3 \pm 0.1 \mathrm{nA}$ and mean value of E-MNs: $1.6 \pm 0.1 \mathrm{nA})$ and the input resistance (mean value of F-MNs: $20.8 \pm 1.9 \mathrm{~m} \Omega$ and mean value of E-MNs: $19.9 \pm 1.9 \mathrm{~m} \Omega$ ).

\section{F-MNs demonstrate a type II profile of firing only}

Figure 1 illustrates the firing responses induced by injection of steps and ramps of current in F-MNs $(n=20)$. Current intensity that induced cell discharge during the depolarizing part of the step protocol did not induce firing during the same repolarizing step intensity (Fig. 1C). Furthermore, for each depolarizing step, the cell demonstrated a firing adaptation (Fig. 1D). Similarly, a depolarizing current ramp followed by the symmetric repolarizing current ramp (Fig. $1 \mathrm{~A}$ ), confirmed the early derecruitment with an average ePIC of $0.5 \pm 0.01 \mathrm{nA}$ and revealed a clockwise hysteresis $(F-I$ plot, Fig. $1 B$ ). In 5 of the 20 F-MNs, the intensity of the recruiting and derecruiting currents were equal among some of the 3 trials. However, even for these particular F-MNs, the $F-I$ relationship showed the same clockwise hysteresis with a shape corresponding to the type II profile (Fig. $1 B$ ) and an average depolarizing slope of $20.4 \pm 0.8 \mathrm{~Hz} / \mathrm{nA}$. 


\section{E-MNs demonstrate multiple firing profiles}

In contrast with the pool of F-MNs, E-MNs showed heterogeneous profiles because the four types of firing could be observed and showed average of ePIC $0.05 \pm$ $0.02 \mathrm{nA}$ and average depolarizing slope of $12.3 \pm 0.8 \mathrm{~Hz} / \mathrm{nA}$.

In 3 of $23 \mathrm{E}-\mathrm{MNs}$, the intensity of the current that induced firing during depolarizing steps was equal to the one that stopped discharge during repolarizing steps (type I profile) (Fig. $2 C, n=2$ ). Moreover, the firing frequency was identical in ascending and descending steps that induced spiking and no firing adaptation could be seen during these two steps (Fig. $2 D)$. The ramp protocol confirmed that the intensity of injected current was equal for recruitment and derecruitment (Fig. $2 A, n=3)$. Furthermore, the $F-I$ plot showed a superimposed linear relationship of the firing frequency (Fig. $2 B$ ).

Thirteen of 23 E-MNs (not illustrated) had a repetitive firing similar to that of F-MNs (type II profile) which will not be further described here.

A type III or type IV profile, corresponding to a self-sustained discharge pattern on the repolarizing part of the protocol was observed in 7 of 23 E-MNs. These motoneurons kept firing during the steps of repolarizing current, whereas they were previously silent during the corresponding similar steps of depolarizing current (Fig. 2I,J;n=2). This suggests that PICs were activated during this protocol.

Type III and IV profiles displayed the same sustained firing during the step protocol. The current ramp protocol allowed us to investigate whether motoneurons display both profiles or not. For instance, type III profile could be identified in 5 of 23 E-MNs because the intensity of the derecruiting current (repolarizing ramp) was lower than that of the recruiting current (depolarizing ramp) (Fig. 2E) and the firing frequency overlapped on both portions of the ramp (Fig. $2 F$ ).

In addition to producing a self-sustained discharge, motoneurons with a type IV profile showed a long depolarization after the end of the firing (Fig. $2 G ; n=2$ ). Furthermore, the membrane potential at the end of the protocol remained more depolarized than the resting membrane potential. The corresponding $F-I$ relationship showed a counterclockwise hysteresis shape (Fig. $2 \mathrm{H}$ ).

\section{Comparative analysis in $\mathrm{E}-\mathrm{MNs}$ versus $\mathrm{F}-\mathrm{MNs}$}

Figure $3 A$ summarizes the distribution of the different firing profiles observed in the two populations of interest. All F-MNs responded in a similar way ( $n=20,100 \%$ type II profile), whereas the responses of E-MNs varied from early derecruitment to selfsustained discharge. Overall, $13 \%$ of E-MNs $(n=3)$ showed type I profile, $57 \%$ type II profile $(n=13)$ and $30 \%$ type III/IV profile $(n=7)$.

We investigated whether PICs are likely to all be absent from a sample of 20 motoneurons randomly chosen among a population of 43 cells containing 7 motoneurons expressing PICs (Rozanov, 1936). The number of ways of selecting 20 motoneurons out of a batch of 43 equals the number of combinations taking 20 things at a time out of 43 , and is just $N=43 ! /(20$ ! 23!). By hypothesis, these combinations are all equiprobable. Let $A$ be the
B

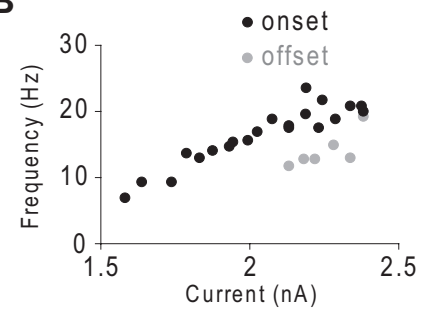

D

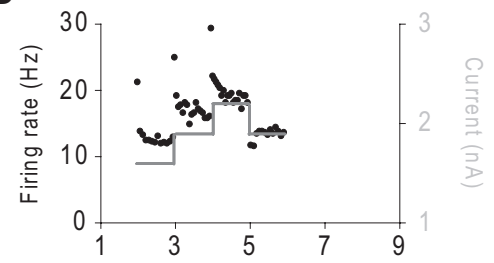
Then $A$ occurs whenever all 20 motoneurons belong to the set of $36(=43-7)$, no-PIC-expressing motoneurons. Hence the number of combinations favorable to $A$ is $N(A)=36 ! /(20 ! 16 !)$. The probability of the event $A$ equals $P(A)=N(A) / N=(36 ! 20$ ! $23 !) /(20$ ! $16 ! 43 !)=0.007$. Thus, considering that the whole motoneuron population is homogeneous, the probability to record no PICs in 20 motoneurons randomly sampled is $p<0.007$. This makes the results very unlikely to happen by chance and clearly supports a difference between F-MNs and E-MNs.

With regard to the amplitude of antidromic spikes, a significant difference was observed between E-MNs and F-MNs having a type II profile (Fig. $3 B$; $p<0.05$; one-way ANOVA). No difference in the resistance was observed, whereas a significant difference was measured between the rheobases of E-MNs having a type II and a type III/IV profile (Fig. $3 B ; p<0.05$; one-way ANOVA). Significant differences between the flexor population and groups of E-MNs were also found for the ePIC and the $F-I$ slope (Fig. 3B; respective mean value of ePIC and F-I slope, Flexor type II $0.46 \pm 0.04 \mathrm{nA} ; 20.4 \pm 0.8 \mathrm{~Hz} / \mathrm{nA}$; Extensor type I $0.05 \pm 0.01 \mathrm{nA} ; 13.04 \pm 0.9 \mathrm{~Hz} / \mathrm{nA}$; Extensor type II $0.12 \pm 0.03$ $\mathrm{nA} ; 11.5 \pm 0.8 \mathrm{~Hz} / \mathrm{nA}$; Extensor type III-IV $-0.12 \pm 0.03 \mathrm{nA}$; $13.6 \pm 2.6 \mathrm{~Hz} / \mathrm{nA})$.

\section{Discussion}

In the present experiments, much care was taken to apply a stimulation protocol designed to efficiently activate PICs. In fact, a depolarizing ramp of current with a slope that is too fast can be inefficient even in neurons expressing the appropriate channels. To avoid this situation, we first tested different slopes and durations of depolarization, previously described in the literature as having the strong potential to efficiently elicit PICs (Button et al., 2006). Furthermore, bias depolarizing currents, known to promote PICs were applied with a particular attention in neurons "without" PICs without any success, suggesting that PICs could not be activated in such motoneurons under our experimental conditions. Current clamp recordings allowed us to record both passive and active properties of the cell, such that we showed the difference between the firing profiles of F-MNs and E-MNs. 
A

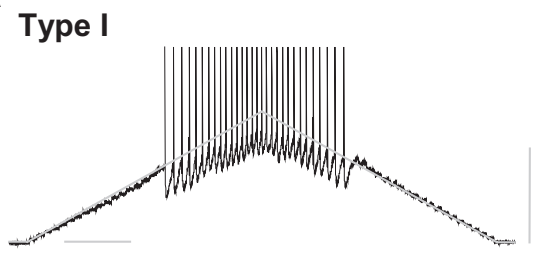

C

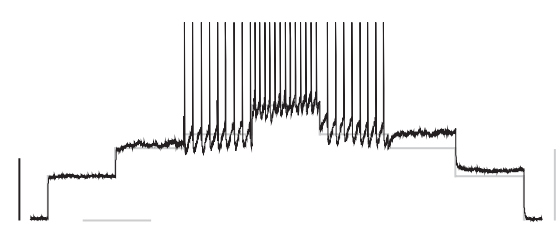

E

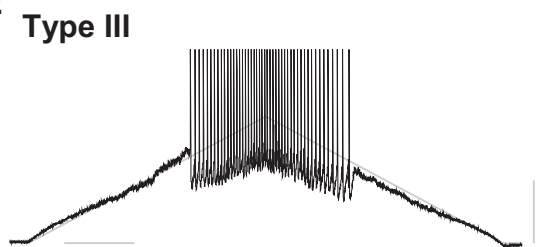

G

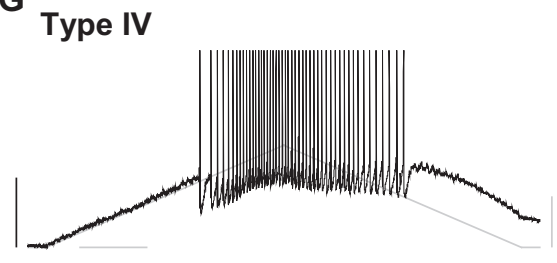

I

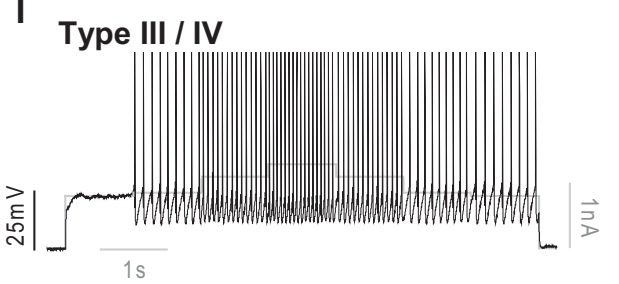

B

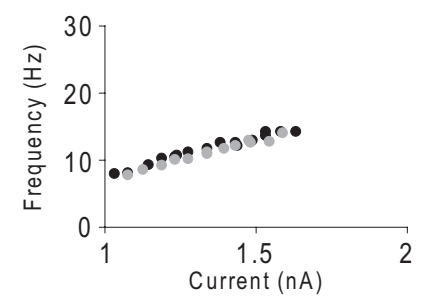

D

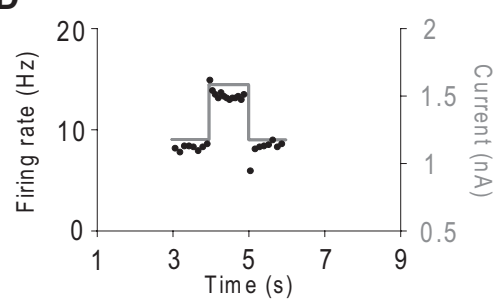

$\mathbf{F}$

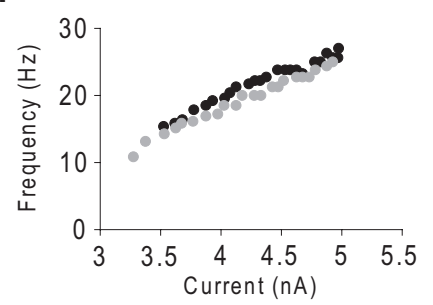

H

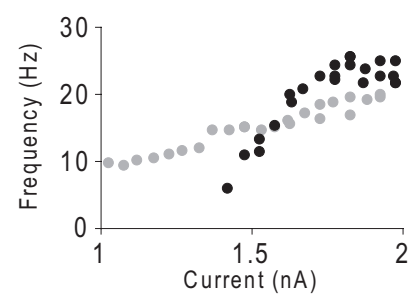

$\mathbf{J}$

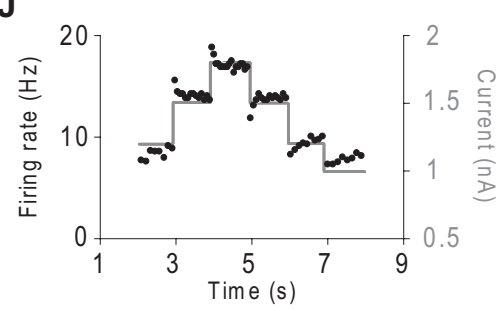

Figure 2. Multiple discharge pattern (type I-IV) in E-MNs. $\boldsymbol{A}, \boldsymbol{C}, \boldsymbol{E}, \boldsymbol{G}, \boldsymbol{I}$, Different membrane potential responses (black) to both step and triangular shaped current (gray). $\boldsymbol{B}, \boldsymbol{D}, \boldsymbol{F}, \boldsymbol{H}, \boldsymbol{J}$, Plot of the $F-I$ relationship of the data shown in $\boldsymbol{A}, \boldsymbol{C}, \boldsymbol{E}, \boldsymbol{G}$, and $\boldsymbol{I}$, respectively. $\boldsymbol{A}$ and $\boldsymbol{C}$ illustrate a motoneuron with a type I profile, in which the injected current intensities that start and stop the firing are equal. $F-/$ relationship demonstrates an overlap of the firing rates during ascending and descending current ramps ( $\boldsymbol{B}$ ) or steps $(\boldsymbol{D})$. $\boldsymbol{E}$ and $\boldsymbol{F}$ illustrate a motoneuron with a type III profile showing a self-sustained firing and an overlap of the $F-I$ curve. The type IV profile shown in $\boldsymbol{G}$ and $\boldsymbol{H}$ is characterized by a self-sustained firing followed by a depolarized state. $F$-I relationship demonstrates a counterclockwise hysteresis $(\boldsymbol{H})$. E-MNs showing type III and IV profiles demonstrate a wind-up following the step-shaped current protocol $(\boldsymbol{I}, \boldsymbol{J})$. Note that a type II profile was also observed in E-MNs.

The triangular protocol used in our study has been used by many others in motoneurons of various animal models such as cat, mice, rat and turtle. Although different discharge patterns were described, no correlation between the cellular firing pattern and the function of the innervated muscle has ever been described. An attempt was made in adult cats to correlate the type of motor unit (FF: fast fatigable; FR: fast resistant; S: Slow) to the level of bistability of lumbar motoneurons (Lee and Heckman, 1998a,b). However, the recordings were only made in E-MNs. They showed that the $S$ and FR types are correlated to a fully bistable property and that the FF type is only correlated to partial bistability. This finding is coherent with our data, in which several types of discharge pattern were recorded in rat E-MNs. In another previous study, identified motoneurons innervating posterior biceps or pretibial flexors were recorded (Hounsgaard et al., 1988b). The authors suggested a "dominance of extensor motoneurons among motoneurons which show bistable properties in decerebrate cat." We now provide evidence that in the newborn rat, only E-MNs have the capacity to develop bistable properties.

In most studies, neuromodulators are shown to facilitate PICs (Hounsgaard et al., 1988b; Li et al., 2007). In the in vitro sacrocaudal spinal cord preparation of adult rat, monoamine receptor antagonists inhibit sodium PICs (Harvey et al., 2006a,b), indicating the presence of an endogenous source of monoamines. In our preparation in which fast synaptic transmission was blocked, the endogenous release of monoamines in the lumbar spinal cord cannot be excluded, because the brainstem descending system was preserved and could contribute to release monoamines at the spinal level. Such endogenous release of monoamines could then facilitate PICs in E-MNs displaying the type III/IV firing profiles. The pharmacological blockade of such monoamine receptors could help understanding this further. Nevertheless, only $30 \%$ of E-MNs were classified as showing the type III/IV profiles. The early developmental stage could account for this. First, at such stage (P4-P7), the descending pathways have not completely invaded the lumbar enlargement (Tanaka et al., 1992), especially the monoamines projections, including the serotonin pathway, which are implicated in bistability (Hounsgaard et al., 1988b; Vinay et al., 2000b). Second, it is known that some motoneuronal properties, such as repetitive firing, are immature at this stage and are still evolving during the following weeks (Vinay et al., 2000b). Whether adding serotonin to the bath or recording motoneurons at more mature stages may increase the number of E-MNs displaying the type III/IV profiles is unknown and requires further investigation.

In contrast to E-MNs, our data indicate that during the first postnatal week, F-MNs are not able to demonstrate PIC activation. A possible explanation is that $5-\mathrm{HT}_{2}$ receptors, known to be implicated in PICs facilitation (Harvey et al., 2006a), are more expressed in E-MNs than in F-MNs in the rat spinal cord (Vult von Steyern and Lømo, 2005). Furthermore, during the first 
postnatal week, maturation of the discharge pattern is faster in F-MNs than in E-MNs (Vinay et al., 2000a). This suggests that F-MNs should be able to display selfsustained discharge earlier than E-MNs. Nevertheless, we cannot rule out that F-MNs do not express PICs and that at later developmental stages they are only able to produce a type II profile. Indeed, experiments were performed during the first postnatal weeks where dramatic changes occur before acquisition of a normal adult profile (Vinay et al., 2000a). F-MNs could be able to generate PICs after a facilitation by neuromodulators. As suggested before, adding monoamine to the preparation could unmask the other firing profiles in F-MNs. For example, L-DOPA administration could unmask plateaus in motoneurons of adult spinalized cats (Conway et al., 1988). Whether the PIC differentiation between flexor and extensor is transient, it could be linked to the rapid maturation of motor behavior that takes place during the first postnatal week. Interestingly, the pups become able to support their body weight at the end of the first week (Jamon and Clarac, 1998), that is at the end of the period of time we investigated. The activation of PICs between the fourth and seventh postnatal days, specifically in E-MNs, could actively participate to sustained activity in antigravity muscles imposed by development of postural activity. The PIC expression in flexors could come later in the development to create a wider range of movements.

In the adult rat (Goudard et al., 1992), like in other adult models [cats (Hounsgaard et al., 1988b)], PICs are not recorded in all motoneurons. From a functional point of view, the more simple explanation will be that the motoneurons not expressing self-sustained discharges might be more important in the pool of F-MNs, and would explain the different patterns of activity recorded in muscles of adult animals. At rest, PICs would be useful in motoneurons showing a tonic firing pattern involved in the control of static postures. During locomotion, it would help patterning the E-MNs activity, whose activation is necessary during the stance phases that are prolonged whereas F-MNs, active during the short swing phase, do not fire long lasting bursts (Goudard et al., 1992). However, further works will be necessary to compare intrinsic properties in adult motoneurons. Because membrane properties such as PICs play an essential role in generating the locomotor pattern (Zhong et al., 2007) these findings could provide important insights into our understanding of the generation of locomotion.

\section{References}

Bennett DJ, Hultborn H, Fedirchuk B, Gorassini M (1998a) Synaptic activation of plateaus in hindlimb motoneurons of decerebrate cats. J Neurophysiol 80:2023-2037.

Bennett DJ, Hultborn H, Fedirchuk B, Gorassini M (1998b) Short-term plasticity in hindlimb motoneurons of decerebrate cats. J Neurophysiol 80:2038-2045.
Bennett DJ, Li Y, Siu M (2001) Plateau potentials in sacrocaudal motoneurons of chronic spinal rats, recorded in vitro. J Neurophysiol 86:1955-1971.

Button DC, Gardiner K, Marqueste T, Gardiner PF (2006) Frequencycurrent relationships of rat hindlimb alpha-motoneurones. J Physiol 573:663-677.

Conway BA, Hultborn H, Kiehn O, Mintz I (1988) Plateau potentials in alpha-motoneurones induced by intravenous injection of L-dopa and clonidine in the spinal cat. J Physiol 405:369-384.

Goudard I, Orsal D, Cabelguen JM (1992) An electromyographic study of the hindlimb locomotor movements in the acute thalamic rat. Eur J Neurosci 4:1130-1139.

Halbertsma JM (1983) The stride cycle of the cat: the modelling of locomotion by computerized analysis of automatic recordings. Acta Physiol Scand Suppl 521:1-75.

Harvey PJ, Li X, Li Y, Bennett DJ (2006a) 5HT2 receptor activation facilitates a persistent sodium current and repetitive firing in spinal motoneurons of rats with and without chronic spinal cord injury. J Neurophysiol 96:1158-1170

Harvey PJ, Li X, Li Y, Bennett DJ (2006b) Endogenous monoamine receptor activation is essential for enabling persistent sodium currents and repetitive firing in rat spinal motoneurons. J Neurophysiol 96:1171-1186.

Hounsgaard J, Kiehn O (1989) Serotonin-induced bistability of turtle motoneurones caused by a nifedipine-sensitive calcium plateau potential. J Physiol 414:265-282.

Hounsgaard J, Kiehn O, Mintz I (1988a) Response properties of motoneurones in a slice preparation of the turtle spinal cord. J Physiol 398:575-589.

Hounsgaard J, Hultborn H, Jespersen B, Kiehn O (1988b) Bistability of alpha-motoneurones in the decerebrate cat and in the acute spinal cat after intravenous 5-hydroxytryptophan. J Physiol 405:345-367. 
Jamon M, Clarac F (1998) Early walking in the neonatal rat. A kinematic study. Behav Neurosci 112:1218-1228.

Kiehn O, Eken T (1998) Functional role of plateau potentials in vertebrate motor neurons. Curr Opin Neurobiol 8:746-752.

Lee RH, Heckman CJ (1998a) Bistability in spinal motoneurons in vivo: systematic variations in rhythmic firing patterns. J Neurophysiol 80:572-582.

Lee RH, Heckman CJ (1998b) Bistability in spinal motoneurons in vivo: systematic variations in persistent inward currents. J Neurophysiol 80:583-593.

Li X, Murray K, Harvey PJ, Ballou EW, Bennett DJ (2007) Serotonin facilitates a persistent calcium current in motoneurons of rats with or without chronic spinal cord injury. J Neurophysiol 97:1236-1246.

Pflieger JF, Clarac F, Vinay L (2002) Postural modifications and neuronal excitability changes induced by a short-term serotonin depletion during neonatal development in the rat. J Neurosci 22:5108-5117.

Rozanov Y (1936) Probability theory: a concise course. Ed. Dover [translated to English by Silverman R]. New York: Dover.
Russell DF, Hartline DK (1978) Bursting neural networks: a reexamination. Science 200:453-456.

Tanaka H, Mori S, Kimura H (1992) Developmental changes in the serotoninergic innervation of hindlimb extensor motoneurons in neonatal rats. Brain Res Dev Brain Res 65:1-12.

Vinay L, Brocard F, Clarac F (2000a) Differential maturation of motoneurons innervating ankle flexor and extensor muscles in the neonatal rat. Eur J Neurosci 12:4562-4566.

Vinay L, Brocard F, Pflieger JF, Simeoni-Alias J, Clarac F (2000b) Perinatal development of lumbar motoneurons and their inputs in the rat. Brain Res Bull 53:635-647.

Vult von Steyern F, Lømo T (2005) Postnatal appearance of 5-HT2A receptors on fast flexor and slow extensor rat motor neurons. Neuroscience 136:87-93.

Zhong G, Masino MA, Harris-Warrick RM (2007) Persistent sodium currents participate in fictive locomotion generation in neonatal mouse spinal cord. J Neurosci 27:4507-4518. 